\title{
Use of Steroids and Immunosuppressive Drugs in the Management of Posterior Uveitis
}

\author{
SUSAN LIGHTMAN
}

London

\begin{abstract}
Summary
Posterior uveitis can occur in all age-groups and often has devastating effects on vision. The visual loss can have a variety of causes including cataract formation, vitritis, optic nerve damage and macular oedema. In patients with active inflammatory disease resulting in reduced vision, steroids are still the best drug but cyclosporin is becoming widely used in combination with steroid theraphy, in patients who do not respond to steroids alone or in whom the dose of steroids required to control the disease process is unacceptably high.

Cyclosporin works specifically on activated T-lymphocytes and does not therefore result in bone marrow suppression like the more conventional immunosuppressive drugs.

This paper discusses the indications for treatment in patients with posterior uveitis and how to use steroids, cyclosporin and other immunosuppressive drugs in the management of this condition.
\end{abstract}

Many patients with posterior uveitis are healthy in all other respects and do not develop a systemic disease whereas others have ocular inflammation as part of a multiorgan disease process. Examination of enucleated eyes from patients with posterior uveitis of many differing aetiologies, has shown that whatever the clincial picture, the predominant infiltrating cell type in the affected ocular tissues is the T-lymphocyte. ${ }^{1}$ Autoimmune mechanisms are thought to play an important role in these disease processes whether they are localised to the eye or occurring as part of a multisystem disorder.

The initiating factors in all these immunemediated disorders are largely unknown but are thought to be a complicated mixture of genetic, immunologic and viral factors. ${ }^{2}$ It is likely that the initiating events determine the site and type of pathological process within the eye since the perpetuation mechanisms appear to be similar-at least in that they are mediated by the same sub-type of T lymphocytes. Management of the inflammatory component of these diseases is directed at suppressing the body's hyperactive immune response rather than aiming for a cure. Suppression needs to be continued until the disease process becomes 'burnt out' and therefore ceases to result in tissue damage.

\section{Indications for Immunosuppressive \\ Treatment}

Inflammation in the posterior segment of the eye can have devastating effects on vision via a variety of mechanisms. These include amongst others the development of cataract, glaucoma and retinal detachment, in addition to vitritis, retinal, disc and macular oedema. It is obvious from this that these patients need detailed ophthalmic assessment to determine the cause or causes of visual loss so that the 
correct form of treatment can be initiated. Often, there is a combination of factors and the relative contribution of each needs to be determined. In particular, in patients with significant cataract, particularly of the posterior sub-capsular type, it can often be difficult to determine the presence or absence of macular oedema with accuracy. Fluerescein angiography with or without fluoroscopy, can be very useful in this situation as it is often easier to see dye leakage at the posterior pole than to visualise that area by biomicroscopy in the presence of medial opacities (Fig. 1).

It is obvious that only those patients in whom there is an active inflammatory process which may cause visual loss require immunosuppressive treatment. Since we are unable to cure the primary disease process, current treatment is aimed at suppression of the overactive immune system and should only be initiated if really necessary. Not all patients with posterior uveitis have sight threatening disease - in some it can be very mild with a peripheral periphlebitis and/or a mild vitritis giving rise to the symptoms of floaters alone. In others, the disease process may be mild for a period of time and then worsen. Treatment should not be given unless necessary since its sequelae in terms of systemic side effects can be worse than those of the ocular pathology. In particular, vitreous floaters, due to cellular and inflammatory debris, without associated posterior pole oedema or other sight threatening disease, should not be an indication for the commencement of immunosuppressive therapy.

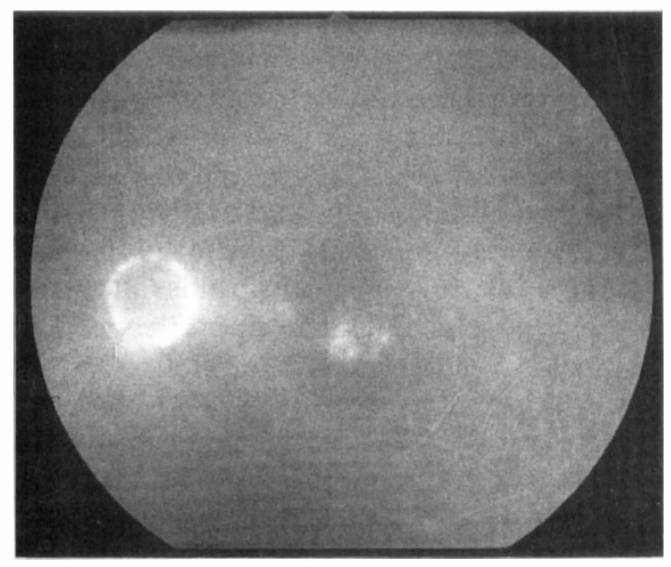

Fig. 1. Fluorescein angiogram showing cystoid macular oedema in a patient with lens opacities.
The next major determinant of the type of treatment is whether there is unilateral or bilateral ocular involvement. Some patients already have a situation in which one eye has pathology which is non-reversible-i.e. a macular hole or ischaemic retina or exacavated optic disc. Treatment is then directed at the other eye if it is involved in the inflammatory process and in this group of patients, together with those who only have uniocular involvement, it may be possible to avoid the problems of high dose systemic therapy altogether by administering steroids locally into the orbit.

\section{Corticosteroids}

Corticosteroids remain the most useful and

effective drugs for the management of the chronic inflammatory process. These drugs work by a variety of mechanisms on various stages of the immune and inflammatory response ${ }^{3}$ and are accompanied by systemic side effects, which can limit their usefulness.

\section{Orbital Floor Steroids}

When uniocular treatment is considered appropriate for the reasons outlined above, depomedrone given locally as an orbital floor injection can be very useful. ${ }^{4}$ We have not found that the site of injection within the orbit to be of importance and use the orbital floor route rather than trying to aim the steroid depot to lie near the region of the posterior pole. It is ideal to give the full dose of $40 \mathrm{mg}$ in $1 \mathrm{ml}$ since if less is given and the patient does not respond, one is left with the question-did it not help because the dose of steroid given was too low or are steroids by this route not going to be effective and the patient requires systemic therapy? It can take two to four weeks before a response is seen as judged by clinical examination including the visual acuity, and unless there is another pressing reason for further intervention, patients should be given adequate time to respond.

\section{Systemic Steroids}

Unfortunately, not all patients respond to orbital floor steroid injections, and together with those who have bilateral ocular involvement, treatment with systemic steroids is necessary. Again, these patients should be 
given adequate doses of the steroids to suppress the inflammatory response and answer the question - can the sequelae of the chronic inflammatory process in this patient be reversed with steroids? In some patients, the posterior pole oedema is unresponsive to all forms of treatment, presumably indicating irreversible damage to the blood-retinal barrier and there is no further point subjecting these people to all the problems associated with long term steroid and other immunosuppressive therapy.

The starting dose of steroids should be 40 $80 \mathrm{mg} /$ day according to disease severity, appropriately reduced according to age and weight for children under the age of 12 years. Alternate day therapy should be avoided at the initiation of therapy since patients seem to take longer to respond and often notice a decrease in vision on non-therapy days. Patients who develop indigestion whilst on these high doses of tablets can be helped by antacids or by administration of histamine $\mathrm{H}_{2}$ receptor antagonists.

Slow reduction of the steroid dose is advocated since the only option available if the disease process ceases to be under adequate control, is to raise the steroid dose back to the higher level. Efficacy of therapy is judged by improvement of the clinical signs as well as by an increase of visual acuity when vitritis and posterior pole oedema are the reasons for its reduction. The dose is gradually reduced until the inflammatory process ceases to be adequately controlled or until the drugs can be tailed off and discontinued when the disease is no longer active.

\section{Other Immunosuppressive Drugs}

Many patients can be satisfactorily managed with steroids alone with the dose being reduced to an acceptable maintenance level (usually less than $15 \mathrm{mg} /$ day). However, there are situations in which the inflammatory process can only be controlled with unacceptably high levels of steroids - any attempt at steroid dose reduction being accompanied by an increase in disease activity. These patients require the introduction of additional immuno-suppressive drugs to facilitate control of the disease on a lower dose of steroids. The conventional immunosuppressive drugs azathioprine, chlorambucil and cyclophosphamide can be used but cyclosporin is rapidly becoming the drug of choice for this role in patients of all ages providing they have normal renal function. ${ }^{5}$

\section{Cyclosporin}

Cyclosporin works in a different manner from the more conventional immunosuppressive drugs in that it is specific for activated T-lymphocytes. ${ }^{6}$ It now plays a major role in the management of organ transplant rejection ${ }^{7}$ in addition to other autoimmune diseases. ${ }^{6}$ The T-cell, subtype CD4+, which is found infiltrating the eye in posterior uveitis as well as in target organs in other autoimmune diseases, secretes lymphokines, which are soluble factors having a variety of diverse biological functions, when it becomes activated. ${ }^{8}$ Cyclosporin inhibits the secretion of some of these lymphokines ${ }^{6}$ and there is a resultant decrease in inflammatory activity. ${ }^{9}$

Unfortunately at the dose at which cyclosporin is effective when used alone $(10 \mathrm{mg} / \mathrm{kg} /$ day), it has permanent damaging effects on the kidneys which now preclude its use. ${ }^{10}$ Other side effects of cyclosporin include tremor, gastrointestinal upsets (which are often transient) hirsutism and hepatotoxicity. No cases of lymphoma have been reported. ${ }^{11}$ At the lower safer dose of $5 \mathrm{mg} / \mathrm{kg} /$ day the side effects are less marked but, it is ineffective on its own in the initial management of posterior uveitis and has to be used in combination with systemic steroids. Exactly how these drugs are used in combination varies from centre to centre but a useful regime has been to increase the steroids back to $40 \mathrm{mg}$ / day for at least a week prior to the introduction of cyclosporin at a dose of $5 \mathrm{mg} / \mathrm{kg} /$ day in two divided doses and then to slowly reduce the steroid dose. In some patients, it has been possible to tail off the steroids altogether when the disease is quiescent, leaving the patient controlled on a small dose of cyclosporin.

The cyclosporin dose is mainly controlled by the serum creatinine-should the latter rise, the dose of cyclosporin is reduced. The blood pressure also needs careful monitoring. Patients with a raised serum creatinine prior to treatment should not be started on cyclos- 
porin. Trough levels of cyclosporin in the blood can sometimes be useful with the blood therapeutic and toxic range depending on the type of assay used by the laboratory. ${ }^{12}$ If the addition of cyclosporin allows the steroid dose to be reduced to a lower level than prior to its introduction, then the aim of its use has been achieved. If not, then it is pointless to continue its use and other immunosuppressive drugs need to be tried.

It is important to be aware that reduction and termination of cyclosporin therapy can be associated with a rebound increase in inflammatory activity. ${ }^{13}$ This needs to be anticipated so that the steroid dose is increased or other immunosuppressive drugs are added while the cyclosporin is being reduced.

\section{Other Immunosuppressives}

Azathioprine is the least toxic of the classic immunosuppressive drugs with its major effect being on the bone-marrow where the lymphocytes are generated. It is useful in the long term management of patients with posterior uveitis although we have found it to be less useful in the management of acute disease. Many patients are transferred to steroid and azathioprine therapy when the ocular inflammation has been brought under control with steroids and cyclosporin. As these patients often need treatment for severalyears, many go through cycles of different drugs at different stages of ocular inflammatory activity.

Many patients with ocular Behçet's disease used to be given chlorambucil in addition to steroids ${ }^{14}$ but many centres now use cyclosporin instead to avoid the effects of sterility and in the longer term, of lymphoma. Further long term comparative studies are needed in these patients to assess the relative usefulness of cyclosporin and azathioprine in Behçet's disease.

Both cyclophosphamide and chlorambucil are contraindicated in children with uveitis because of the long term sequelae whereas cyclosporin can be used and we have found it to be very effective.

\section{Conclusions}

Using adequate immunosuppressive therapy, many patients with posterior uveitis can be helped. Steroids remain the most useful drug but their side effects at high doses are profound. The major recent advance in therapy has been in the use of cyclosporin to control the inflammation but it too is limited by its side effects - particularly on the kidney. Long term studies are underway on the use of steroids and low dose cyclosporin to monitor its renal effects.

We await the development of the next generation of cyclosporin type drugs-ideally these should be lymphocyte specific and not require the concomitant use of systemic steroids. That they should be free of side effects remains a dream but in reality, should be safer than those we currently use.

Key words: cyclosporin, immunosuppression, oedema, steroids, uveitis.

\section{References}

${ }^{1}$ Lightman $\mathrm{S}$ and Chan C: Immune mechanisms in choroidoretinal inflammation. Eye 1990, 4: 345-53.

${ }^{2}$ Cohen I and Wekerle $\mathrm{H}$ : Autoimmunity, self-recognition, and blocking factors. In 'Autoimmunity: Genetic, Immunologic, Virologic and Clinical Aspects': ed Talal N. 1977, Academic Press NY.

${ }^{3}$ Rang HP and Dale MM: Glucorcorticoids. In: 'Pharmacology' eds Rang and Dale p 394-402. Churchill Livingstone.

${ }^{4}$ Russin MM, Tessher HH, Cunha-Vas JG: Posterior subtenon injection of corticosteroids in uveitis patients with cystoid macula oedema. Jpn J Ophthalmol 1988, 34: 385-91.

${ }^{5}$ Towler H, Cliffe A, Whitting P, Forrester J: Low dose cyclosporin A therapy in chronic posterior uveitis. Eye 1989, 3: 282-87.

${ }^{6}$ Borel JV and Ryffel B: The mechanism of action of cyclosporin: A continuing puzzle. In: Schindler, R. ed. Cyclosporin in Autoimmune Diseases. Berlin: Springer-Verlag, 1986, pp. 24-31.

${ }^{7}$ Calne RY: Immunosuppression for organ-grafting: Observations on cyclosporin A. Immunol Rev 1979, 46: 113-28.

${ }^{8}$ Thompson AW and Webster LM: The influence of cyclosporin A on cell mediated immunity. Clin Exp Immunol 1988, 71: 369-76.

${ }^{9}$ Nussenblatt RB, Palestine AG, Chan CC: Cyclosporin-A therapy in the treatment of intra-ocular inflammatory disease resistant to systemic corticosteroids and cytotoxic agents. Am J Ophthalmol 1983, 93: 275-81.

${ }^{10}$ Palestine AG, Austin HA, Barlow JE, Autonouych TT, Sabins SG, Prems HE, Nussenblatt RB: Renal histopathologic alterations in patients treated with cyclosporin for uveitis. $N$ Eng J Med 1986, 314: 1293-8.

${ }^{11}$ Van Eraffenried B and Krupp B: Side effects of cyclosporin in renal transplant recipients and in pateints with autoimmune disease. Transplant Proc 1986, 18: 876-83.

${ }^{12}$ Shaw L: Critical issues in cyclosporin monitor- 
ing: Report of the task force on cyclosporin monitoring. Clin Chem 1987, 33: 1629-88.

${ }^{13}$ Binder AI, Graham EM, Sanders MD, Dinning W, James WG, Denman AM: Cyclosporin A in the treatment of severe Behçet's disease. Brit J Rheumatol 1987, 26: 285-91.

${ }^{14}$ Tabbara KF: Chlorambucil in Behçet's disease: A reappraisal. Ophthalmology 1983, 90: 906-8. 